\title{
Modelling and computer simulation of nanostructured devices
}

\author{
Kurapati Srinivas, Duggirala Krishna Rao \\ Nanotechnology Group, Department of Physics, GMR Institute of Technology, Rajam-532127, A.P India
}

Email address:

srinkura@gmail.com (K. Srinivas), srinivas.k@gmrit.org (K. Srinivas)

\section{To cite this article:}

Kurapati Srinivas, Duggirala Krishna Rao. Modelling and Computer Simulation of Nanostructured Devices. American Journal of Nanoscience and Nanotechnology. Vol. 2, No. 3, 2014, pp. 40-44. doi: 10.11648/j.nano.20140203.12

\begin{abstract}
Theory, modeling, and simulation provide investigative tools that support nanotechnology. Multi scale modeling is essential for exploring the numerous, new technology possibilities that research in nanoscience is creating. The purpose of the proposed review work is to explore models and techniques for designing and controlling nanostructured devices and establish links between physical (quantum) device modelling, experimental system identification, model verification and quantum control. Having reliable mathematical and computational models, methods and using standard tools with efficient computer simulation, it is essential to design complicated quantum devices such as semi-conductor nanostructures, superconducting devices before they are fabricated experimentally. The proposed review work will be useful to know the current status of nanostructure devices simulations and the work that is carried out in various centers to understand the physics and transport phenomena occurring in the nanoscale and better design of nanostructured devices.
\end{abstract}

Keywords: Computer Simulations, Nanostructures, Molecular Dynamics, Grid Computers

\section{Introduction}

Theory, modeling, and simulation provide investigative tools that support nanotechnology. To support nanotechnology in the next decade, these tools must be enhanced to solve larger problems, and to do so more accurately and efficiently. Multi scale modeling is essential for exploring the numerous, new technology possibilities that research in nanoscience is creating. It is essential for identifying promising ideas, for developing them into real technologies, and it will be essential for nano manufacturing. Faster computers and better computational methods will be important, but the real challenge is to develop new conceptual and modeling frameworks. New conceptual models must be developed to relate atomistic physics and chemistry to nanoscale structure and then nanoscale structure to the performance of complex materials and integrated nanosystems

The purpose of the proposed review work is to explore models and techniques for designing and controlling nanostructured devices and establish links between physical (quantum) device modelling, experimental system identification, model verification and quantum control. Having reliable mathematical and computational models, methods and using standard tools with efficient computer simulation, it is essential to design complicated quantum devices such as semi-conductor nanostructures, superconducting devices before they are fabricated experimentally. The techniques and models are needed to design, simulate and control the operation of these nano devices consists of Dynamic control simulations require control system models, conventionally based on partial differential equations derived from fundamental principles, such as the control dependent Schroedinger or quantum Liouville equation. Efficient simulation algorithms for physically accurate computational models are crucial for this task. Statistical models simply describing the behavior of an actual device may sometimes be sufficient, or at least be used to augment the differential equation approach to consider specific material properties and the complete behavior of real devices. The proposed review work will definitely useful not only for better theoretical understanding of the physics and transport phenomena occurring in the nano-scale and for the experimental researchers for optimization and better design of nanostructure In most of the cases, the experimental study of Nanotechnology involves high cost for Laboratory set-up and the experimentation processes were also slow. So, one cannot rely on experimental nanotechnology alone. As such, the Computer-Based molecular simulations and modeling 
are one of the foundations of computational nanotechnology. The computer based modeling and simulations were also referred as computational experimentations. In real experiments, the investigator doesn't have full control over the experiment. But, in Computational experimentation the investigator have full control over the experiment. The accuracy of such Computational nano-technology based experiment generally depends on the accuracy of the following things: Intermolecular interaction, Numerical models and Simulation schemes used. Once the accuracy of the Computational Scheme is guaranteed one can use that to investigate various nonlinear interactions whose results are completely unexpected and unforeseen. Apart from it, numerical modeling and computer based simulations also help to understand the theoretical part of the nano-science involved in the nano-system. They allow us to develop useful analytic and predictive models. The computer based simulation techniques [1] are widely used for computational nano-technology.

The frequently used simulation approaches are Monte Carlo (MC) and Molecular Dynamics (MD) methods. The other various simulation methods come from these two basic methods. Apart from it, the optimization techniques were also needed in MC and MD. The computer- based simulation methods, developed for nano- systems, generally consist of a computational procedure performed of few atoms or molecules confined in a small geometrical space. This geometrical space in which the simulation is performed is termed as cell. In the subsequent section, a brief classification of simulation methods based on Accuracy, Computational Time etc were given. After it, classifications of optimizations in molecular simulations were discussed. In next section after it, we have discussed the result of computer -based simulation.

\section{Monte Carlo and Molecular Dynamics Simulation Methods [2]}

The two basic used simulation approaches are Monte Carlo (MC) and Molecular Dynamics (MD) methods. All the other various simulation methods come from these two basic methods. A brief over-view with areas of application of the both are discussed below. These concepts are essentially required to understand the methodology of classification of Computer-Based Simulation methods based on accuracy and time-complexity.MC method uses random numbers to perform calculations. There are many areas of application of MC Methods including nanomaterial. Some important areas where we apply $\mathrm{MC}$ method are: (i) Estimation of large-dimensional integrals (ii) Generating thermodynamic ensembles in order to compute thermal averages of physical equilibrium quantities of interest and simulation of non-equilibrium phenomena such as growth and (ii) Computation of distribution functions out of equilibrium known as Kinetic Monte Carlo [5]. MD deals with predicting the trajectories of atoms subject to their mutual interactions and eventually an external potential. Some important areas of application of MD are: (i) Computation of transport properties such as response functions, viscosity, elastic moduli and thermal conductivity (ii) Thermodynamic properties Local Optimization Methods: (i) Steepest Descent Methods (ii) Damped Newtonian Dynamics (iii) Conjugate Gradient Method (iv) Quasi- Newton Method parallelism. Using these four levels we demonstrate that an almost ideal scaling of the wall time upto 32768processors with a parallel efficiency of $86 \%$ is reached in the simulation of realistically extended and gated field-effect transistors. Obtaining the current characteristics of these devices is reduced to some hundreds of seconds instead of days on a small cluster or months on a single CPU.

\section{Immersive Visualisation}

Interactive visualisation in an immersive environment has crucial requirement on frame rate (normally should $\geq$ $15 \mathrm{fps}$ ). To achieve real-time walkthrough, A. Nakano and R. K. Kalia [10] used a multi resolution MD algorithm and the associated data structures to visualise large quantity atoms. In another paper, A. Nakano [11] employed an octree data structure for visibility culling and levels-ofdetail control for fast rendering. Theoretically, these techniques will decrease the number of polygons to be displayed. However, accounting for the user perspective requires additional calculations. In my opinion, solving large-scale MD visualisation problem requires an integrated systems approach. On a high level, the following four issues are worth mentioning:

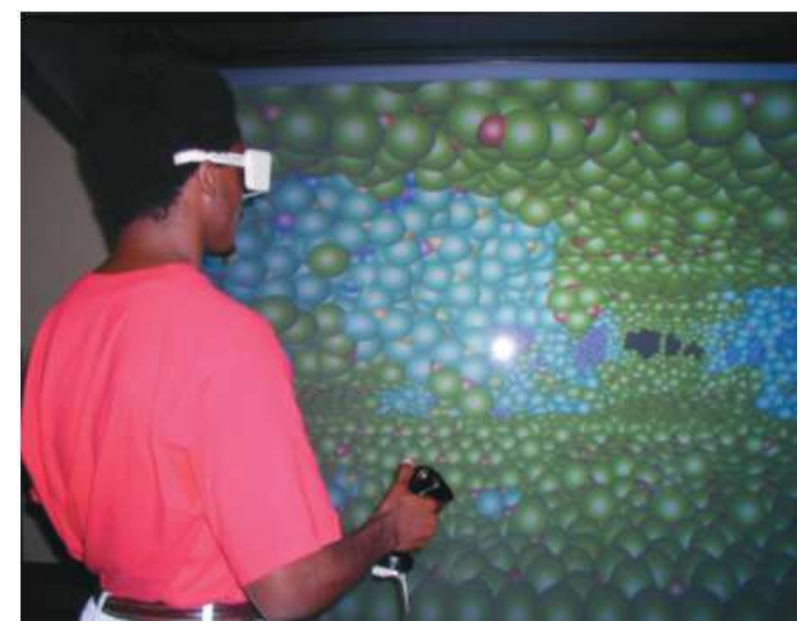

Figure 1. A scientist immersed in an atomistic model of a fractured ceramic nanocomposite

1. Data pre-processing and feature extraction. Generally, a direct rendering of all atoms is not helpful. We are interested in extracting the physically based features that we specify. We can represent these features, if successfully extracted, in an economical way for interactive visualisation. 
2. Scalable parallel visualisation. (as shown in Fig.1) To visualise large-scale MD simulations at the highest possible resolution, we need the processing power and memory of a parallel computer. PC clusters, which have become increasingly popular and affordable, make parallel visualisation an even more attractive approach [12].

3. Optimization of basic graphics functionality. For molecular visualisation, only very few types of objects in massive quantities, such as spheres (as atoms), cylinders (as bonds), and points (as charge density), need to be rendered. Therefore, very efficient graphics routines might be written and optimized.

4. Visualisation management. There exist some useful techniques for large-scale visualisation, such as visibility culling using octree data structure [13], Level of Detail (LOD) and database paging [14], etc. We adopt an integrated approach. Before visualisation, MD simulation results are pre- processed and the coordination number of each atom is calculated. According to the purpose of MD simulations, atoms might be divided into three groups according to their significance, i.e. important, normal and less important. For important atoms, we use high quality spheres (more polygons) to represent them. We also extend the LOD technique for navigation. During fast navigation, atoms are rendered in a lower resolution. One notable phenomenon is that all these systems confine the user to a $2 \mathrm{D}$ environment to visualise and interact with $3 \mathrm{D}$ molecular structures [1].

\section{Computer Simulation of Nanoparticles Formation, Moving, Interaction and Self-Organization [3]}

The method for calculating the moving, interaction and self organization of nanoparticles is offered. The method is based on the potential built up with the help of the approximation of the numerical calculation results using the method of molecular dynamics of the pair wise static interaction of nanoparticles. With the potential of the pair wise interaction of the nanoparticles, which takes into account forces and moments of forces, the method for calculating the ordering and self organizing processes, has been developed. The numeric simulation by the methods of molecular mechanics and dynamics were used for investigating the regularities of the amorphous phase formation and the nucleation and spread of the crystalline or hypo crystalline phases over the entire nanoparticle volume depending on the process parameters, nanoparticles sizes(as shown in Fig.2) and thermodynamic conditions of the ambient. Hemodynamics has four main advantages over the molecular dynamics method because the type of the nanosystem unit cell is different (in molecular dynamics, it is an atom, and it is a nanoparticle in mesodynamics):

1. The number of the variables of a nanosystem reduces because the analysis of the nanoparticle movement is used instead of the calculation of the movement of separate atoms.

2. The number of the bonds between separate nanosystem elements reduces due to a decrease in the quantity of the "nearest neighbours" in the region of the interaction of the nanosystem unit cells.

3. The total computation dimensions of the nanosystem increases due to the calculation unit cell coarsening.

4. The analysis scope for the nanosystem computation extends in time because the time step of the integration of equations increases.

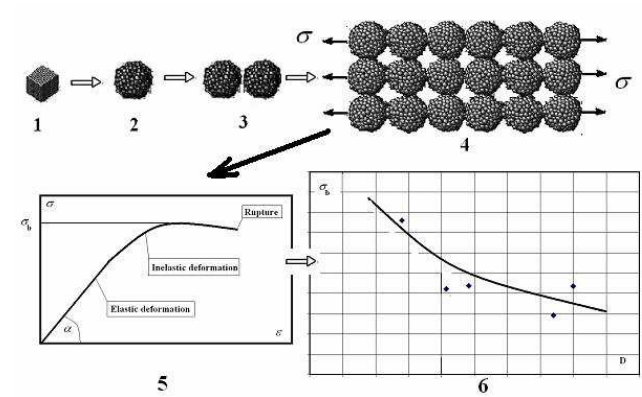

Figure 2. Sequence of calculation of nanoparticle system (1-4) and calculation results $(5,6)$

\section{Geographically Distributed Pc Clusters in the US and Japan [4]}

We have demonstrated the scalability of multiscale MD/QM simulations on a Grid of geographically distributed PC clusters in the US and Japan. The resulting high efficiency (despite the weak communication link) suggests that this is an efficient collaboration mode in computational research. Such a multiscale simulation collaboratory will consist of geographically distributed application specialists who not only maintain their computational modules with most updated simulation algorithms but also provide compute servers that are best suited to their own application codes.

We have shown that the additive multiple-clustering scheme is effective in Gridifying such multiscale simulation codes. Furthermore, the additive hybridization scheme allows multiple layers of nested hybridization including, e.g., MD, DFT, and higher accuracy QM calculations such as configuration interaction. These methods are currently used by separate groups of scientists to solve similar problems at different levels of accuracy and problem sizes (as shown in Fig.3). The multiscale simulation collaboratory on a Grid will allow these scientists to jointly solve challenging scientific/engineering problems. The Gridification approach in this paper should be applicable to other modular multiscale simulations as well. An example is the black-box hybridization scheme based on the multilevel Newton method, in which the residual equation is formulated in a coupled model space combining, e.g., electrostatic and mechanical problems. 
High Performance Computing Cluster Cluster installations primarily comprise two types of standards-based hardware components servers and networking interconnects. Clusters are divided into two major classes high-throughout computing clusters and high-performance computing clusters. High-throughput computing clusters usually connect a large number of nodes using low-end interconnects.

We have implemented multifarious aspects of nano simulation using multi scale modeling on various HPC (High Performance Computing) setups. Distribution of jobs from macro to nano scale has been shown which holds the essence of simulation at nano scale. This distribution is substantiated on MPI (Message Passing Interface) and PVM (Parallel Virtual Machine) on MATLAB, Linux and WCCS (Windows Compute Cluster Server) environments. In this paper we have shown the connections and a novel way of implementing multi scale computations on an HPC setup. We have also compared the implementation of MPI and PVM based HPC setup for MATLAB, Linux and WCCS environments.(Table2)

Table 1. configuration of various hpc setups

\begin{tabular}{|c|c|c|c|}
\hline HPC Setup & Operating System & Math Library & Comm Library \\
\hline \multirow{4}{*}{ MPI } & Microsoft & Extreme & \multirow{4}{*}{ MPI.NET } \\
\hline & Windows Server & Optimization & \\
\hline & 2003 Compute & Numerical & \\
\hline & $\begin{array}{l}\text { Cluster Edition } \\
\text { 64bit }\end{array}$ & $\begin{array}{l}\text { Library for } \\
\text {.NET }\end{array}$ & \\
\hline \multirow{2}{*}{ PVM } & Open SUSE-11.0- & C, parallel & \multirow{2}{*}{$\begin{array}{l}\text { Pvm- } \\
3.4 .5 . x 86 \quad 64\end{array}$} \\
\hline & x86_64 64bit & math library & \\
\hline \multirow{3}{*}{ MATLAB } & \multirow{3}{*}{ Windows Vista } & MATLAB & Distributive \\
\hline & & Inbuilt & computing \\
\hline & & Functions & toolbox \\
\hline
\end{tabular}

Table 2. configuration of various hpc setups

\begin{tabular}{|c|c|c|c|}
\hline HPC Setup & Operating System & Math Library & Comm. Library \\
\hline \multirow{4}{*}{ MPI } & Microsoft & Extreme & \multirow{4}{*}{ MPI.NET } \\
\hline & Windows Server & Optimization & \\
\hline & 2003 Compute & Numerical & \\
\hline & $\begin{array}{l}\text { Cluster Edition } \\
\text { 64bit }\end{array}$ & $\begin{array}{l}\text { Library for } \\
\text {.NET }\end{array}$ & \\
\hline \multirow{2}{*}{ PVM } & Open SUSE-11.0- & C, parallel & \multirow{2}{*}{$\begin{array}{l}\text { Pvm- } \\
\text { 3.4.5.x86_64 }\end{array}$} \\
\hline & x86_64 64bit & math library & \\
\hline \multirow{3}{*}{ MATLAB } & \multirow{3}{*}{ Windows Vista } & MATLAB & Distributive \\
\hline & & Inbuilt & computing \\
\hline & & Functions & toolbox \\
\hline
\end{tabular}

Our prime motive is to characterize the current options available for HPC based setups for multi scale simulations in the field of Nanotechnology. The approach in this direction has to be carried out in view of the requirements of the users. Table 1 gives us the HPC libraries used and the respective OS platforms and math libraries being employed[5] High Performance computing clusters connect more powerful compute nodes using faster interconnects. High-performance computing clusters are more suitable for tightly coupled parallel needed to be performed in an HPC setup applications, which have substantial communication and synchronization requirements. For parallel jobs, the job efficiency also affects resource utilization. To achieve high resource utilization for parallel jobs, both job efficiency and advanced scheduling are required. Efficient job processing depends on effective application design

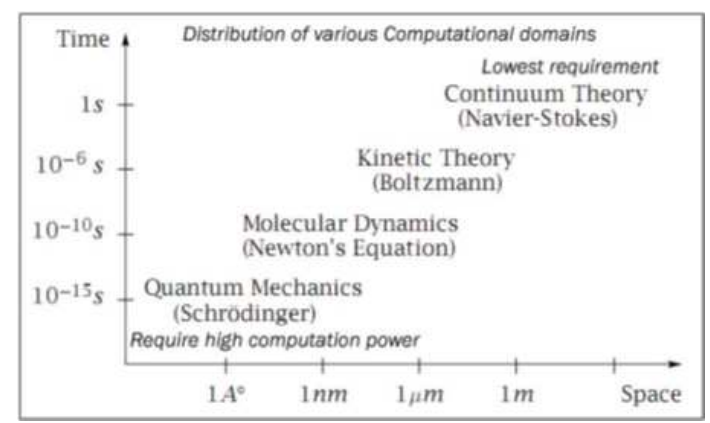

Fig 3. Distribution of various computations.

Technical specifications

- 1 Head Node \& 15 Client Nodes

- 2 x Intel(R) Xeon(R) CPU E5630@2.53GHz

- 24 GB RAM

- 1 TB Hard Disk

Connectivity

- $\quad$ InfiniBand Switch (MTS3600)

- 36 InfiniBand ports

- $\quad$ Up to $40 \mathrm{~Gb} / \mathrm{s}$

- $\quad$ Up to $3.5 \mathrm{~W}$ per port for active cable or fiber module support

\section{Major Nanosimulaton Tools}

1. NanoFEM platform http://www.gospa.ws/en/projects/nanofem-platform/ A Software Platform for Nanoscale Device Simulation and Visualization -- NanoFEM Platform

2. Nano HUB http://www.nanohub.org Online Simulation and More For Nanotechnology A resource for nanoscience and technology, the nanoHUB was created by the NSF-funded Network for Computational Nanotechnology.

3. NanoTCAD ViDES http://vides.nanotcad.com NanoTCAD ViDES is a device simulator able to compute transport in nanoscale devices, and it is particularly devoted to the assessment of the performance of graphene based transistors. In particular, it self-consistently solves the Poisson equation (both 2D and 3D) together with quantum transport equation within the NEGF formalism.

4. NEMO3D

http://cobweb.ecn.purdue.edu/ gekco/nemo3D

5. QuantumWise http://www.quantumwise.com/

- First-principles simulation software for nanoscience

- $\quad$ Atomistix ToolKit (ATK)

- Virtual NanoLab (VNL)

6. nextnano http://www.nextnano.de/

- next generation 3D nanodevice simulator

7. Cybernet http://www.cybernet.co.jp/english/products 
- Optical design and evaluation system

- $\quad$ Electronic design / MEMS

- Nanotechnologies solution

\section{Conclusions}

The purpose of the proposed review work is to explore models and techniques for designing and controlling nanostructured devices and establish links between physical (quantum) device modelling, experimental system identification, model verification and quantum control. Having reliable mathematical and computational models, methods and using standard tools with efficient computer simulation, it is essential to design complicated quantum devices such as semi-conductor nanostructures, superconducting devices before they are fabricated experimentally. Having reliable mathematical and computational models, methods and using standard tools with efficient computer simulation, it is essential to design complicated quantum devices such as semi-conductor nanostructures, superconducting devices before they are fabricated experimentally. The techniques and models are needed to design, simulate and control the operation of these nano devices. High Performance Computing Cluster installations primarily comprise of standards-based hardware components servers and networking interconnects are essential for nano-simulation work. A complete survey is made regarding simulation tools that are necessary for nano-simulation. This paper gives a complete information regarding nano-simualation that helps researchers who wish to work in the field of computational nanotechnology.

\section{Tools for Materials}

Simulations

- NWChem (5.1):

- Abinit (5.4.4):

- $\quad$ CPMD (3.11.1):

LAMMPS (2007): LAMMPS is a classical molecular dynamics code that models an ensemble of particles in a liquid, solid, or gaseous state. It can model atomic, polymeric, biological, metallic, granular, and coarse-grained systems using a variety of force fields and boundary conditions VASP:- VASP is a complex package for performing ab-initio quantummechanical molecular dynamics (MD) simulations. The VASP is based on the (finite-temperature) local-density approximation with the free energy as variational quantity and an exact evaluation of the instantaneous electronic ground state at each MD time step.

NAMD (2.6):

DDSCAT (6.1):

Process Simulator

Centaurs

\section{Acknowledgements}

I would like to acknowledge Prof. C.L.R.S.V. Prasad, Prinipal and Professor Nagendar Parashar Director (Academic) of GMR Institute of Technology, Rajam, A.P, India for their constant encouragement to finish this work.

\section{References}

[1] Mathieu Luisier and Gerhard Klimeck Purdue University - A multi-level parallel simulation approach to electron in transport in nano-scale transistors" (2008). Nanotechnology Publications. Paper 148.

[2] Tamal Sarkar,Samir Chandra Das and Aredhenu Mandal "A study of computer based simulations for Nanosystems and their types" proceeding of Proceedings of 2nd National Conference on Nano - materials and Nanotechnology (21 23 December, 2009), University of Lucknow, U.P

[3] Alexandre V. VakhrouchevComputer simulation of nanoparticles formation, moving, interaction and selforganization Journal of Physics: Conference Series 61 (2007) 26-30

[4] Hideaki Kikuchi et al.Collaborative Simulation Grid: Multiscale Quantum-Mechanical/Classical Atomistic Simulations on Distributed PC Clusters in the US and Japan *0-7695-1524-X/02 2002

[5] IEEERohit Pathak, and Satyadhar Joshi Implementation of Parallelization and Nano Simulation using Multi-Scale Modeling on various HPC setups2009 Conference on Innovative Technologies in Intelligent Systems and Industrial Applications (CITISIA 2009)

[6] Monash University, Sunway campus, Malaysia, 25th \& 26th July 2009.pp249-254. 